Effect of di rection of an external force on crystal I ization of colloi dal particles in a V- shaped groove by sedi ment at i on

\begin{tabular}{|l|l|}
\hline 著者 & Sat o Nasahi de \\
\hline $\begin{array}{l}\text { j our nal or } \\
\text { publ i cat i on ti t l e }\end{array}$ & Japanese Journal of Appl i ed Physi cs \\
\hline vol une & 55 \\
\hline number & 9 \\
\hline page r ange & 095601 \\
\hline year & $2016-09-01$ \\
\hline URL & ht t p: //hdl . handl e. net /2297/46180 \\
\hline
\end{tabular}




\title{
Effect of direction of an external force on crystallization of colloidal particles in a $\mathrm{V}$-shaped groove by sedimentation
}

\author{
Masahide Sato \\ Information Media Center, Kanazawa University, Kanazawa 920-1192, Japan
}

\begin{abstract}
We carried out Langevin dynamics simulations to study the effect of the direction of a uniform external force on the crystallization of colloidal particles in a V-shaped groove. When the inclination of the side walls of a groove was set to a suitable value and the external force bisected the angle, the face-centered-cubic (fcc) structure grew with a $\{100\}$ growth interface. When the external force was inclined, the number of solidified particles decreased with increasing inclination, which is different from the growth in an inverted pyramidal container.
\end{abstract}

\section{Introduction}

The sedimentation of colloidal particles is one of the useful methods of forming a closepacked colloidal crystal. ${ }^{1)}$ When colloidal particles settle on a flat plane, a triangular lattice is formed on the plane to maximize the particle density. Since the face-centeredcubic (fcc) structure is more stable than the hexagonal-close-packed (hcp) structure, ${ }^{2,3}$ ) the triangular lattice acts as the $\{111\}$ face of the fcc structure and a colloidal crystal with the fcc structure grows. However, since stacking faults are easily formed on the $\{111\}$ face of the fcc structure, the random hexagonal-close-packed (rhcp) structure is formed frequently. ${ }^{1,4)}$

van Blaaderen et al. ${ }^{5)}$ used a patterned substrate to avoid the formation of the rhcp structure. They formed a square lattice with a suitable lattice constant on the substrate. Affected by the square lattice, the particles settling on the substrate form a square lattice, which acts as the $\{100\}$ face of the fcc structure. Since the stacking is unique on the $\{100\}$ face of the fcc structure, the formation of the rhcp structure is suppressed and large grains with the fcc structure are formed. The method is called colloidal epitaxy and is a promising method for forming large colloidal crystals. However, to obtain a colloidal crystal with high quality, it is necessary to fabricate the patterned substrate precisely.

Another approach for forming a large colloidal crystal with the fcc structure is to 
grow a colloidal crystal on a pyramidal pit or V-shaped groove. ${ }^{6)}$ In both cases, a triangular lattice acting as the $\{111\}$ plane of the fcc structure is formed spontaneously on the walls of the container. The triangular lattice acts as a substrate for colloidal particles. Affected by the lattice, large grains with the fcc structure are formed.

When a colloidal crystal is grown in a pyramidal pit, the growth interface is directed toward the $\langle 100\rangle$ direction if the external force causing the sedimentation of particles is parallel to the center axis. Owing to the unique stacking on the $\{100\}$ face, grains with the hcp structure hardly appear, irrespective of the particle size, when the apex angle of the pyramidal pit is suitable. The hcp-structured grains, which act as defects in the fcc-structured crystal, increase in number with increasing deviation of the apex angle ${ }^{7,8)}$ but their number hardly depends on the inclination of the force direction. ${ }^{7)}$

In the growth of colloidal particles in a V-shaped groove, ${ }^{6)}$ the growth interface is directed toward the $\langle 110\rangle$ direction if the external force bisects the angle between the two walls of the groove. It is easily expected that the number of defects increases with increasing deviation of the angle between the walls from a suitable value. However, the dependence of defect formation on the force direction may be different from that in the case of growth in a pyramidal pit. Thus, we carried out Langevin dynamics simulations and studied how the force direction affects the crystallization of colloidal particles during growth in a V-shaped groove. In Sect. 2, we introduce the model we used. In Sect. 3, we show the results of simulations. In Sect. 4, we summarize the results and give a brief discussion.

\section{Model}

We assume that the motion of the $i$ th particle obeys the following equations:

$$
\begin{aligned}
m \dot{\boldsymbol{v}}_{i} & =-\zeta \boldsymbol{v}_{i}+\boldsymbol{F}_{\mathrm{ext}}+\sum_{i \neq j} \boldsymbol{F}_{i j}+\boldsymbol{F}_{i}^{B}, \\
\boldsymbol{v}_{i} & =\dot{\boldsymbol{r}}_{i},
\end{aligned}
$$

where $m, t$, and $\boldsymbol{r}_{i}$ represent the mass of a particle, time, and the position of the $i$ th particle, respectively. In Eq. (1), the first term in the right-hand side represents the viscous force, which is proportional to the particle velocity. The second term is the external force expressed as $\boldsymbol{F}_{\text {ext }}=F_{\text {ext }} \boldsymbol{e}_{\text {ext }}$, where $F_{\text {ext }}$ and $\boldsymbol{e}_{\text {ext }}$ represent the magnitude of the force and a unit vector in the force direction, respectively. The third term is the sum of the internal forces from the other particles. We assume that the internal force between the $i$ th and $j$ th particles is given by the gradient of the Weeks-Chandler-Anderson 
(WCA) potential ${ }^{9)} U\left(r_{i j}\right)$. The short-range repulsive potential $U\left(r_{i j}\right)$ is defined as

$$
U\left(r_{i j}\right)= \begin{cases}4 \epsilon\left[\left(\frac{\sigma}{r_{i j}}\right)^{12}-\left(\frac{\sigma}{r_{i j}}\right)^{6}+\frac{1}{4}\right] & \left(r_{i j} \leq r_{\text {in }}\right), \\ 0 & \left(r_{i j} \geq r_{\text {in }}\right),\end{cases}
$$

where $r_{i j}=\left|\boldsymbol{r}_{j}-\boldsymbol{r}_{i}\right|, \sigma$ is a characteristic length representing the particle diameter, $\epsilon$ represents the strength of the repulsion and $r_{\text {in }}$ is given by $2^{1 / 6} \sigma$. The fourth term represents the random force caused by thermal noise. We assume that $\boldsymbol{F}_{i}^{B}$ satisfies $\left\langle\boldsymbol{F}_{i}^{\mathrm{B}}\right\rangle=\mathbf{0}$ and $\left\langle F_{k, i}^{\mathrm{B}}(t) F_{l, j}^{\mathrm{B}}\left(t^{\prime}\right)\right\rangle=2 \zeta k_{\mathrm{B}} T \delta_{i j} \delta_{k l} \delta\left(t-t^{\prime}\right)$, where $F_{k, i}^{\mathrm{B}}(t)$ is the $k$ component of the random force acting on the $i$ th particle at time $t$.

In our previous studies, ${ }^{7,8,10-15)}$ we neglected the inertial term $m \ddot{\boldsymbol{r}}_{i}$ for simplicity, but this term may affect the crystallization of particles. Thus, we take account of the inertial term and carry out simulations using a simple Verlet-type algorithm. ${ }^{16}$ The difference equations of Eqs. (1) and (2) are given by ${ }^{16)}$

$$
\begin{aligned}
& \tilde{\boldsymbol{r}}_{i}\left(\tilde{t}_{n+1}\right)=\tilde{\boldsymbol{r}}_{i}\left(\tilde{t}_{n}\right)+b \Delta \tilde{t} \tilde{\boldsymbol{v}}_{i}\left(\tilde{t}_{n}\right)+\frac{b}{2} \gamma(\Delta \tilde{t})^{2}\left[\tilde{\boldsymbol{F}}_{\mathrm{ext}}+\sum_{i \neq j} \tilde{\boldsymbol{F}}_{i j}\left(\tilde{t}_{n}\right)\right]+\frac{b}{2} \gamma \Delta \tilde{t} \tilde{\boldsymbol{F}}_{i}^{\mathrm{B}}\left(\tilde{t}_{n+1}\right) \\
& \tilde{\tilde{v}}_{i}\left(\tilde{t}_{n+1}\right)=a \tilde{v}_{i}\left(\tilde{t}_{n}\right)+\frac{\gamma \Delta \tilde{t}}{2}\left\{(a+1) \tilde{\boldsymbol{F}}_{\mathrm{ext}}+\sum_{i \neq j}\left[a \tilde{\boldsymbol{F}}_{i j}\left(\tilde{t}_{n}\right)+\tilde{\boldsymbol{F}}_{i j}\left(\tilde{t}_{n+1}\right)\right]\right\}+b \gamma \tilde{\boldsymbol{F}}_{i}^{\mathrm{B}}\left(\tilde{t}_{n+1}\right)(5)
\end{aligned}
$$

where the scaled variables $\tilde{\boldsymbol{r}}_{i}, \tilde{t}, \tilde{\boldsymbol{F}}_{\text {ext }}, \tilde{\boldsymbol{F}}_{i}^{\mathrm{B}}$, and $\tilde{\boldsymbol{F}}_{i j}$ satisfy $\boldsymbol{r}_{i}=\sigma \tilde{\boldsymbol{r}}_{i}, t=\sigma^{2} \zeta \tilde{t} / \epsilon, \boldsymbol{F}_{\text {ext }}=$ $\epsilon \tilde{\boldsymbol{F}_{\text {ext }}} / \sigma, \boldsymbol{F}_{i}^{\mathrm{B}}=\epsilon \tilde{\boldsymbol{F}_{i}^{\mathrm{B}}} / \sigma$, and $\boldsymbol{F}_{i j}=\epsilon \tilde{\boldsymbol{F}}_{i j} / \sigma$, respectively. $\Delta \tilde{t}$ is defined as $\Delta \tilde{t}=\tilde{t}_{n+1}-\tilde{t}_{n}$.

The other parameters are defined as

$$
\begin{aligned}
\gamma & =\frac{\zeta^{2} \sigma^{2}}{m \epsilon} \\
a & =\left(1-\frac{\gamma \Delta \tilde{t}}{2}\right)\left(1+\frac{\gamma \Delta \tilde{t}}{2}\right)^{-1}, \\
b & =\left(1+\frac{\gamma \Delta \tilde{t}}{2}\right)^{-1} .
\end{aligned}
$$

The normalized thermal noise for the $i$ th particle, $\tilde{\boldsymbol{F}}_{i}^{\mathrm{B}}\left(\tilde{t}_{n}\right)$, satisfies $\left\langle\tilde{\boldsymbol{F}}_{i}^{\mathrm{B}}\left(\tilde{t}_{n}\right)\right\rangle=\mathbf{0}$ and $\left\langle\tilde{F}_{k, i}^{\mathrm{B}}\left(\tilde{t}_{n}\right) \tilde{F}_{l, i}^{\mathrm{B}}\left(\tilde{t}_{m}\right)\right\rangle=2 \tilde{R}_{B} \Delta \tilde{t} \delta_{k l} \delta_{n m}$ with $\tilde{R}_{B}=k_{\mathrm{B}} T / \epsilon$. When we neglect the inertial term, we obtain a simple difference equation given by ${ }^{17)}$

$$
\tilde{\boldsymbol{r}}_{i}\left(\tilde{t}_{n+1}\right)=\tilde{\boldsymbol{r}}_{i}\left(\tilde{t}_{n}\right)+\left[\tilde{\boldsymbol{F}}_{\mathrm{ext}}+\sum_{i \neq j} \tilde{\boldsymbol{F}}_{i j}\left(\tilde{t}_{n}\right)\right] \Delta \tilde{t}+\Delta \tilde{\boldsymbol{r}}_{i}^{\mathrm{B}}\left(\tilde{t}_{n+1}\right),
$$

where $\left\langle\Delta \tilde{r}_{k, i}^{\mathrm{B}}\left(\tilde{t}_{n}\right) \Delta \tilde{r}_{l, j}^{\mathrm{B}}\left(\tilde{t}_{m}\right)\right\rangle=2 \tilde{R}^{\mathrm{B}} \Delta \tilde{t} \delta_{k l} \delta_{i j} \delta_{n m}$. We also carry out simulations using Eq. (9), and compare the results of the simulations with the results obtained using 
Eqs. (4) and (5).

\section{Results of simulations}

Figure 1 shows a snapshot of the $\mathrm{V}$-shaped container we used in our simulations. The positions of the corners are given by $\mathrm{A}(0,0,0), \mathrm{B}(L, 0, L / \sqrt{2}), \mathrm{C}(-L, 0, L / \sqrt{2}), \mathrm{D}(0,2 L, 0)$, $\mathrm{E}(L, 2 L, L / \sqrt{2})$, and $\mathrm{F}(-L, 2 L, L / \sqrt{2})$, where $L$ is determined so that the volume fraction of particles is 0.1 . The angle $\angle \mathrm{BAC}$ is $-180 \tan ^{-1}(2 \sqrt{2}) / \pi^{\circ}$; thus, the horizontal plane becomes the $\{110\}$ face of the fcc structure if a triangular lattice is formed on the side walls ABED and ACFD.

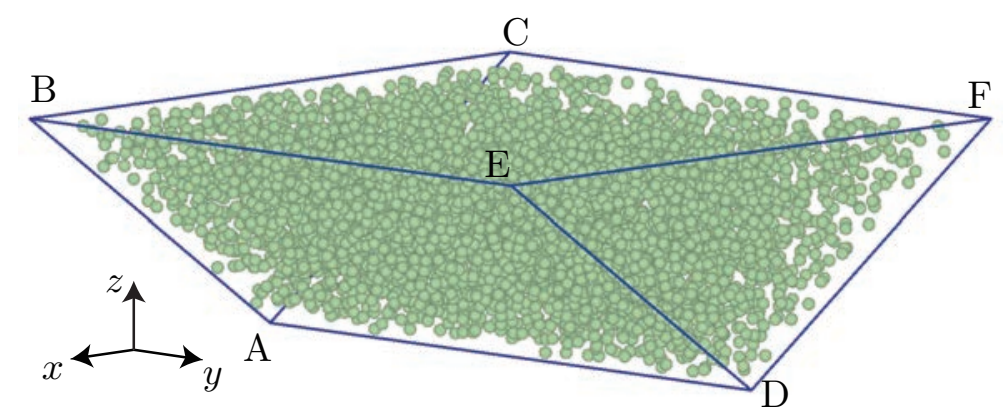

Fig. 1. (color online) Typical shape of a V-shaped groove.

The number of particles is 20000 and $\sigma=1$. Initially, we placed the particles in the container at random and set the velocities of the particles to be 0 . After we moved the particles by a random force with $R_{\mathrm{B}}=0.1$ during $0<\tilde{t}<1000$, we set the time to $\tilde{t}=0$ again and added an external force, which causes the sedimentation of particles. The particles have a radius of 0.5 and act as hard spheres at the walls of the container, so that perfectly elastic collision occurs between the walls and the particles. In our simulations, $\Delta \tilde{t}$ is smaller than $10^{-3}$.

Figure 2 shows snapshots during sedimentation with $\boldsymbol{F}_{\text {ext }}=0.8(0,0,-1)$, which are shown from the $y$-direction. Particles are represented as small circles. The particles are distributed uniformly at an early stage [Fig. 2(a)]. With increasing time, the particles settle and the particle density increases near the side walls [Fig. 2(b)]. First, ordering of the particles in the direction normal to the walls proceeds and layers parallel to the side walls are formed [Fig. 2(c)]. Then, ordering proceeds in each layer and a spotted pattern appears in the lower region [Fig. 2(d)]. Figure 3 shows a snapshot from the $x$-direction. We can see inverted triangular regions at $y=28, y=53$, and $y=80$ (Fig. 3), which are disordered regions remaining in the ordered region. 

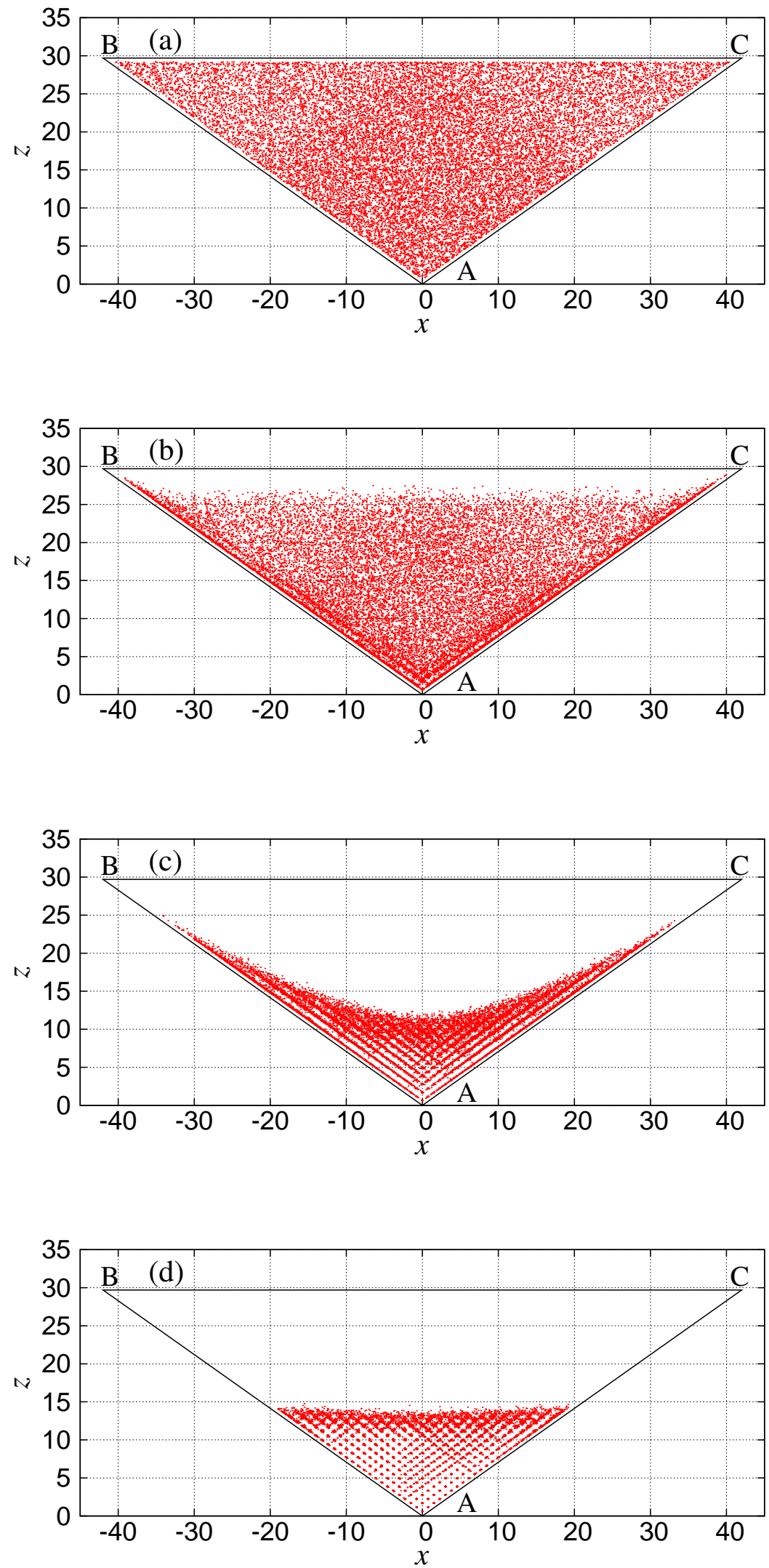

Fig. 2. (color online) Snapshots during sedimentation with $\boldsymbol{F}_{\text {ext }}=0.8(0,0,-1)$, which are shown from the $y$-direction. $\tilde{t}$ is (a) 0 , (b) 5 , (c) 30 , and (d) 250 . 


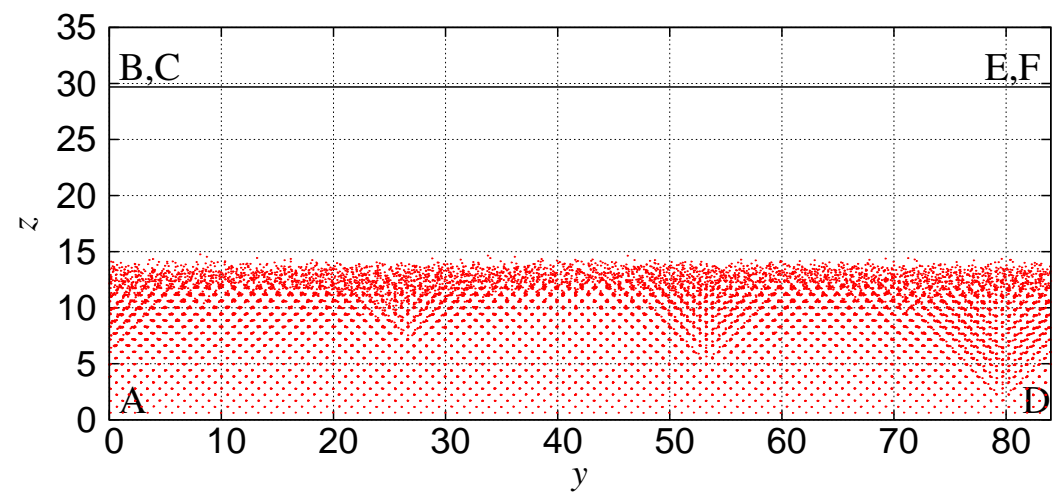

Fig. 3. (color online) Snapshot during sedimentation with $\boldsymbol{F}_{\text {ext }}=0.8(0,0,-1)$ at $\tilde{t}=250$, which is shown from the $x$-direction.

From Figs. 2 and 3, we find that the fcc structure is mainly formed in the container. However, to determine the ordering of the particles more quantitatively, we introduce order parameters $d_{l}(i, j), Q_{l}(i)$, and $w_{l}(i){ }^{4,18,19)}$ The parameter $d_{l}(i, j)$ is used to determine a property of the relation between the $i$ th and $j$ th particles. $d_{l}(i, j)$ is defined by

$$
d_{l}(i, j)=\sum_{m=-l}^{l} q_{l, m}(i) q_{l, m}^{*}(j)
$$

where

$$
q_{l, m}(i)=\frac{1}{n_{\mathrm{n}}} \sum_{j=1}^{n_{\mathrm{n}}} Y_{l}^{m}\left(\theta_{i j}, \phi_{i j}\right),
$$

and $q_{l, m}(i)^{*}$ is the complex conjugate of $q_{l, m}(i)$. In Eq. (11), $n_{\mathrm{n}}$ represents the number of neighbors of the $i$ th particle, $Y_{l}^{m}\left(\theta_{i j}, \phi_{i j}\right)$ is the spherical harmonic, and $\theta_{i j}$ and $\phi_{i j}$ represent the polar and azimuthal angles for $\boldsymbol{r}_{j}-\boldsymbol{r}_{i}$, respectively. When $d_{6}(i, j)>0.7$, we regard the connection between the $i$ th and $j$ th particles as a solid-like one. A particle with four or more solid-like connections is regarded as a solid-like particle.

In our simulations, the hcp and fcc structures are possibly close-packed structures. To distinguish these two structures, we use $Q_{l}(i)$, and $w_{l}(i)$. They are defined as

$$
\begin{aligned}
Q_{l}(i) & =\sqrt{\frac{4 \pi}{2 l+1} \sum_{m=-l}^{l}\left|q_{l, m}(i)\right|^{2}} \\
w_{l}(i) & =\sum_{m_{1}, m_{2}, m_{3}}\left(\begin{array}{ccc}
l & l & l \\
m_{1} & m_{2} & m_{3}
\end{array}\right)\left(\frac{4 \pi}{2 l+1}\right)^{3 / 2} \frac{q_{l, m_{1}}(i) q_{l, m_{2}}(i) q_{l, m_{3}}(i)}{Q_{l}(i)^{3}},
\end{aligned}
$$


where the integers $m_{1}, m_{2}$, and $m_{3}$ satisfy $-l \leq m_{1}, m_{2}, m_{3} \leq l$ and $m_{1}+m_{2}+$ $m_{3}=0$. The first term in the right-hand side of Eq. (13) represents the Wigner $3-j$ symbol. When $n \leq 9$, we may not be able to distinguish the fcc structure from the hcp structure properly. Thus, we regard a solid-like particle with less than nine neighbors as a disordered solid-like particle. We use $Q_{l}(i)$ and $w_{l}(i)$ for the particles with 10 or more neighbors. We regard the local structure around the $i$ th particle as the fcc structure when $-0.18<w_{4}(i)<-0.01$ and $0.175<Q_{4}(i)<0.2$. When $0.02<w_{4}(i)<0.15$ and $0.06<Q_{4}(i)<0.15,{ }^{7,8,10-15)}$ the structure is regarded as the hcp structure. When $w_{4}(i)$ and $Q_{4}(i)$ have other values, we regard the $i$ th particle as a disordered solid.
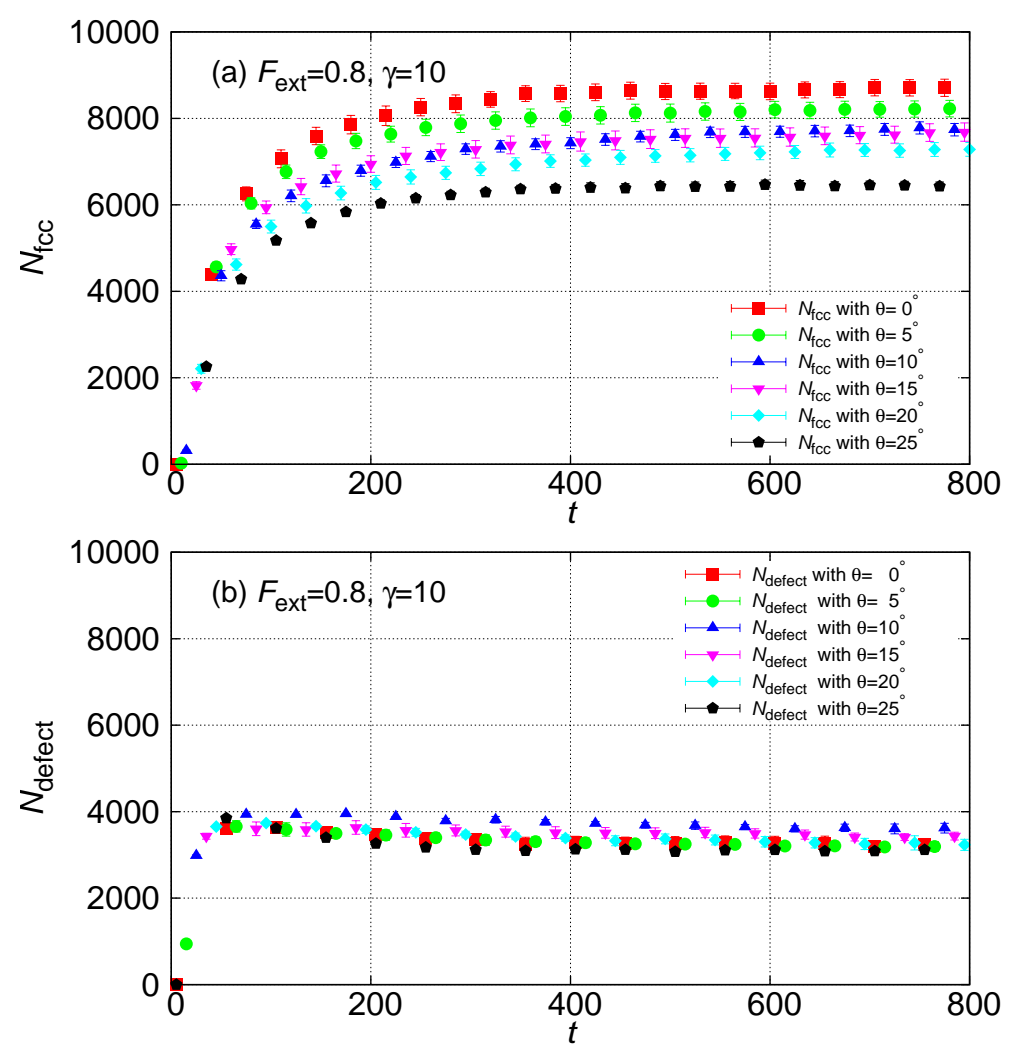

Fig. 4. (color online) Time evolutions of (a) $N_{\text {fcc }}$ and (b) $N_{\text {defect }}$ with $\boldsymbol{F}_{\text {ext }}=0.8$ and $\gamma=10$.

We investigate how the number of particles with the fcc structure, $N_{\text {fcc }}$, depends on $\boldsymbol{F}_{\text {ext }}$. Figure 4(a) shows the time evolutions of the average value of $N_{\text {fcc }}$ with various $\theta$ values. The data are averaged over 10 individual runs. Error bars represent the standard errors. $\theta$ represents the angle between $(0,0,-1)$ and $\boldsymbol{F}_{\text {ext }} . N_{\text {fcc }}$ increases with increasing time at an early stage and finally saturates. The saturated value of $N_{\mathrm{fcc}}$ clearly decreases with increasing $\theta$; thus, the dependence of $N_{\text {fcc }}$ on $\theta$ is different from that in the case of 
solidification in a pyramidal pit. ${ }^{7)}$ Figure 4(b) shows the time evolution of the number of particles acting as defects, $N_{\text {defect }}$, which is the sum of the numbers of hcp-structured particles and disordered solid-like particles. We want to know how many defects there are in the ordered region. Thus, to estimate $N_{\text {defect }}$, we exclude the disordered particles and the particles with the hcp structure, which are on top of the ordered region. $N_{\text {defect }}$ increases in the initial stage. Then, it decreases slightly and saturates at a later stage. Different from $N_{\text {fcc }}$, the saturated value of $N_{\text {defect }}$ hardly depends on $\theta$. From Figs. 4(a) and $4(\mathrm{~b})$, we find that the ratio of $N_{\text {defect }}$ to $N_{\text {fcc }}$ increases with increasing $\theta$.
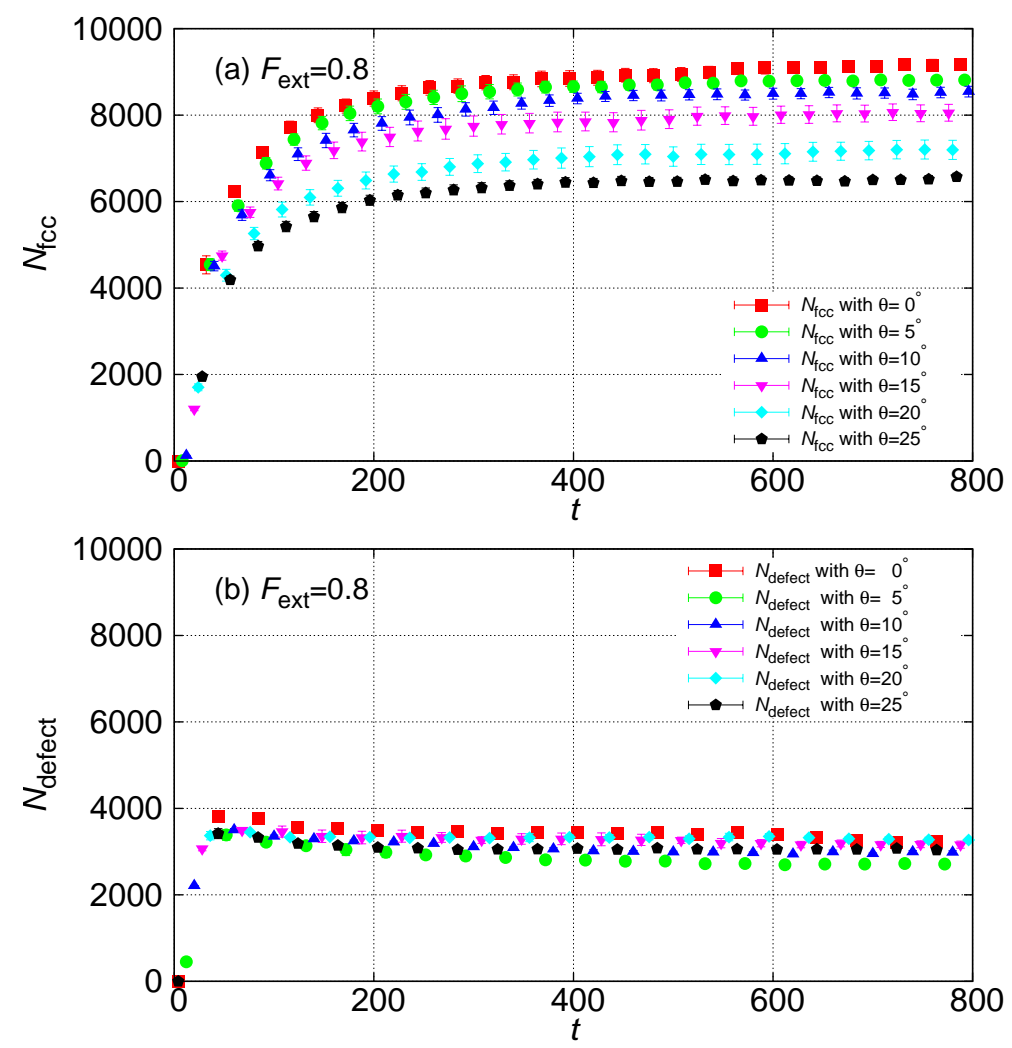

Fig. 5. (color online) Time evolutions of (a) $N_{\text {fcc }}$ and (b) $N_{\text {defect }}$ with $\boldsymbol{F}_{\text {ext }}=0.8$ obtained from Eq. (9).

In the above simulations, we used a larger $\gamma$ so that the inertial term was small. If we carry out simulation with Eq. (9), we will probably obtain similar results. Figure 5 represents the time evolutions of $N_{\text {fcc }}$ and $N_{\text {defect }}$ in a simulation with Eq. (9). Both $N_{\text {fcc }}$ and $N_{\text {defect }}$ are as large as those in Fig. 4 , which agrees with our expectation.

We also carry out a simulation with a small $\gamma$. Figure 6 shows the time evolutions of $N_{\text {fcc }}$ and $N_{\text {defect }}$ with $\gamma=0.1$. These numbers decrease with increasing inclination of 

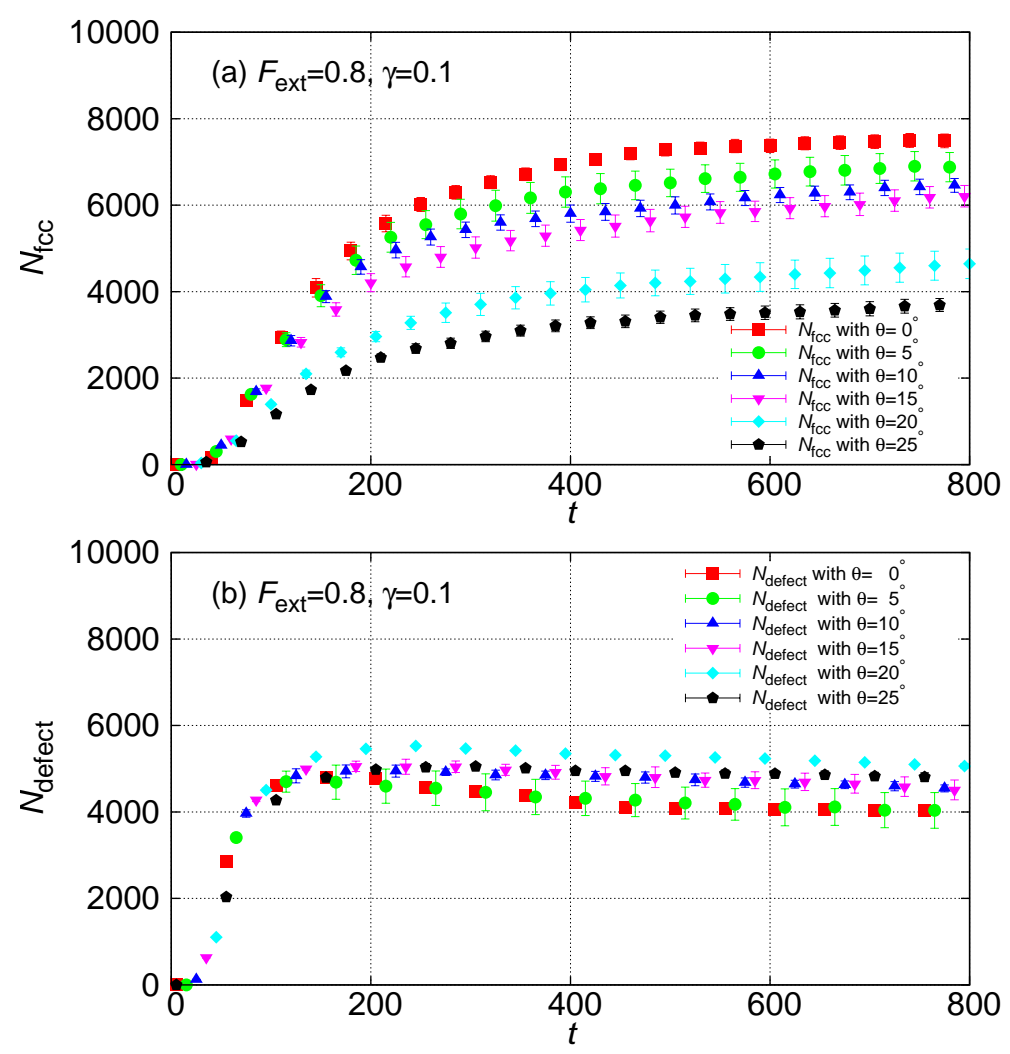

Fig. 6. (color online) Time evolutions of (a) $N_{\text {fcc }}$ and (b) $N_{\text {defect }}$ with $\boldsymbol{F}_{\text {ext }}=0.8$ and $\gamma=0.1$.

the force direction. The tendency is the same as that in the case of solidification with $\gamma=10$. However, $N_{\text {fcc }}$ is smaller and $N_{\text {defect }}$ is slightly larger than those in Fig. 4 . The ratio of the defects increases with decreasing $\gamma$.

Finally, we also investigate how the saturated values of $N_{\text {fcc }}$ and $N_{\text {defect }}$ depend on $\gamma$ and the container shape. Figure 7 shows the dependence of the saturated values of $N_{\text {fcc }}$ and $N_{\text {defect }}$ on $\theta$ with $\boldsymbol{F}_{\text {ext }}=0.8$. When we use Eq. (9) or Eqs. (4) and (5) with $\gamma=10, N_{\text {defect }}$ hardly depends on $\theta$ and $N_{\text {fcc }}$ decreases slightly with increasing $\theta$. When we use Eqs. (4) and (5) with $\gamma=0.1, N_{\text {defect }}$ increases and $N_{\text {fcc }}$ decreases with increasing $\theta$, but the change in $N_{\text {fcc }}$ is much larger than that in $N_{\text {defect }}$. Thus, irrespective of $\gamma$, the change in the ratio of $N_{\text {defect }}$ to $N_{\text {fcc }}$ is mainly caused not by a decrease in $N_{\text {defect }}$ but by an increase in $N_{\text {fcc }}$. Figure 8 shows the dependence of the saturated value of $N_{\text {fcc }}(\theta) / N_{\text {fcc }}(0)$ on the container shape. We carried out simulations using Eq. (9) as the difference equation. The magnitude of the force is given by $\boldsymbol{F}_{\text {ext }}=0.2$, which is smaller than that in previous simulations. The containers are a pyramidal container and a V-shaped groove. In both containers, $N_{\text {fcc }}(\theta) / N_{\text {fcc }}(0)$ decreases with increasing $\theta$. However, the decrease in the ratio in the $\mathrm{V}$-shaped groove is much larger than that in 

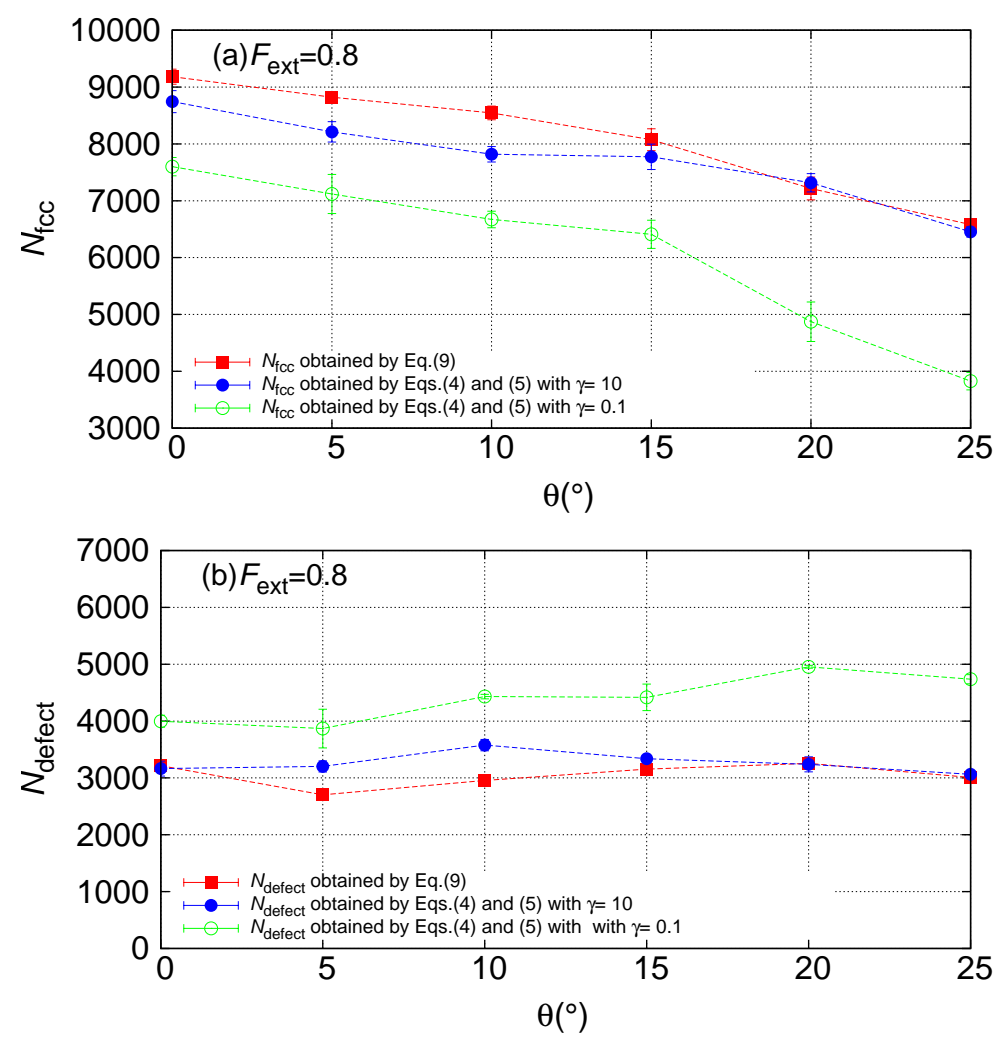

Fig. 7. (color online) Dependence of the saturated values of (a) $N_{\text {fcc }}$ and (b) $N_{\text {defect }}$ on $\theta$ with $\boldsymbol{F}_{\text {ext }}=0.8$.

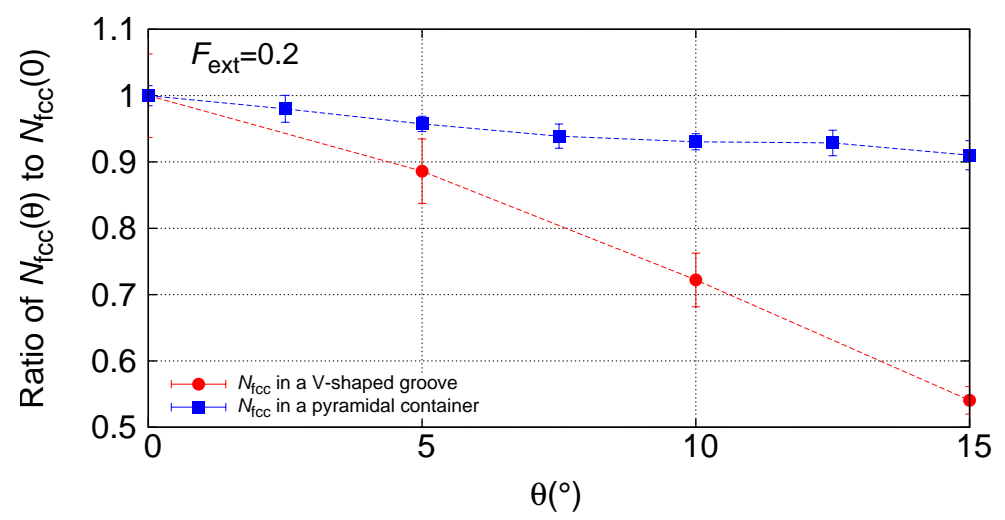

Fig. 8. (color online) Dependence of the saturated value of $N_{\mathrm{fcc}}(\theta) / N_{\mathrm{fcc}}(0)$ on the container form with $\boldsymbol{F}_{\text {ext }}=0.2$, where we use Eq. (9).

the pyramidal container. When the colloidal crystal grows in the V-shaped groove, the effect of the inclination of the force direction on $N_{\text {fcc }}$ is larger than that in the case of growth in the pyramidal container. 


\section{Summary and discussion}

In this paper, we studied the effect of the force direction on the crystallization of colloidal particles by sedimentation. When we used a V-shaped groove with a suitable angle as a container, the fcc structure whose $\{111\}$ face was parallel to the side walls was formed. When the force direction was parallel to the $z$-direction, the growth interface became the $\{100\}$ face and $N_{\text {defect }}$ was small. Different from the results of our previous simulations in a pyramidal container, the ratio of $N_{\text {defect }}$ to $N_{\text {fcc }}$ increased with increasing inclination of the force direction. The main reason was the decrease in $N_{\text {defect }}$. In the case of growth in a pyramidal container, the growing crystal was surrounded by four pyramidal planes. When the force direction was slightly inclined, the layers formed by settled particles were not so thin; thus, the effect of the force direction was small. On the other hand, the growing crystal was surrounded by only two walls in the case of growth in a groove. When the force was tilted, the layers of settled particles spread on one of the walls. The layers were thinner than those obtained in the pyramidal container. Thus, the number of solidified particles decreased more than that in the pyramidal container.

In our simulations, we used a Verlet-type algorithm in which the inertial term was taken into account. When $\gamma \gg 1$, the effect of the inertial term was small and the results of the simulations were similar to those with Eq. (9). When $\gamma \ll 1$, owing to the inertial term, $N_{\text {fcc }}$ decreased. When we used Eq. (9), the motion of particles did not depend on their velocity. The particles moved toward the low-potential direction; thus, a regular structure was formed easily. On the other hand, when $\gamma$ was small, the direction of particle motion was determined not only by the potential decrease but also by the particle velocity; thus, the particles did not always move toward the lowpotential direction. If the particle density was increased quickly by the external force before the particles moved to suitable positions determined by the potential energy, the disordered particles remained in the bulk. The increase in $\gamma$ decreased defect formation in the bulk. Thus, if the particle size is fixed, we should use particles made from a light material to form a high-quality colloidal crystal. The parameter $\gamma$ is expressed as $\gamma=54 \pi \eta \sigma \tilde{R}_{\mathrm{B}} /\left(\rho K_{\mathrm{B}} T\right)$, where $\eta$ is the viscosity and $\rho$ is the particle density. For polystyrene particles in water, $\rho=1.05 \mathrm{~g} / \mathrm{cm}^{3}$ and $\eta=8.51 \times 10^{-4} \mathrm{~Pa} \cdot \mathrm{s}$. When the diameter of the particles is given by $\rho=1 \mu \mathrm{m}, \gamma$ is estimated as $\gamma=2.8 \times 10^{7} \tilde{R}_{\mathrm{B}}$. We could not estimate $\gamma$ precisely because $\tilde{R}_{\mathrm{B}}=k_{\mathrm{B}} T / \epsilon$ is an unknown parameter, but $\tilde{R}_{\mathrm{B}}$ is probably not so large and $\gamma$ is small. Thus, the effect of the inertial term is neglected 
and we can use Eq. (9) to consider the crystallization of polystyrene particles in Ref. 6 . However, if we consider the crystallization of smaller particles with a high density, we need to use the Verlet-type algorithm given by Eqs. (4) and (5).

\section{Acknowledgement}

This work was supported by JSPS KAKENHI Grant Numbers 26390054 and 16K05470. 


\section{References}

1) J. Zhu, M. Li, R. Rogers, W. Meyer, R. H. Ottewill, STS-73 Space Shuttle Crew, W. B. Russel, and P. M. Chaikin, Nature 387, 883 (1997).

2) L. V. Woodcock, Nature 385, 141 (1997).

3) H. Míguez, F. Meseguer, C. López, A. Mifsud, J. S. Moya, and L. Vázquez, Langmuir 13, 6009 (1997).

4) M. Marechal, M. Hermes, and M. Dijkstra, J. Chem. Phys. 135, 034510 (2011).

5) A. van Blaaderen, R. Ruel, and P. Wiltzius, Nature 385, 321 (1997).

6) S. Matsuo, T. Fujine, K. Fukuda, S. Juodkazis, and H. Misawa, Appl. Phys. Lett. 82, 4285 (2003).

7) M. Sato, M. Y. H. Widianto, and Y. Kanatsu, Jpn. J. Appl. Phys. 54, 115503 (2015).

8) Y. Kanatsu and M. Sato, J. Phys. Soc. Jpn. 84, 11460 (2015).

9) J. D. Weeks, D. Chandler, and H. C. Anderson, J. Chem. Phys. 54, 5237 (1971).

10) M. Sato, H. Katsuno, and Y. Suzuki, Phys. Rev. E 87, 032403 (2013).

11) M. Sato, H. Katsuno, and Y. Suzuki, J. Phys. Soc. Jpn. 82, 084804 (2013).

12) M. Sato, Y. Suzuki, and H. Katsuno, J. Cryst. Growth 401, 87 (2014).

13) M. Fujine, M. Sato, H. Katsuno, and Y. Suzuki, Phys. Rev. E 89, 042401 (2014).

14) M. Fujine, M. Sato, T. Toyooka, H. Katsuno, Y. Suzuki, and T. Sawada, Phys. Rev. E 90, 032404 (2014).

15) Y. Kanatsu and M. Sato, Jpn. J. Appl. Phys. 54, 110301 (2015).

16) N. Grønbech-Jensen and O. Farago, Mol. Phys. 111, 983 (2013).

17) D. L. Ermak, J. Chem. Phys. 62, 4189 (1975).

18) P. J. Steinhardt, D. R. Nelson, and M. Ronchetti, Phys. Rev. B 28, 784 (1983).

19) M. D. Rintoul and S. Torquato, J. Chem. Phys. 105, 9258 (1996). 